\title{
The Role of Fever Clinics in the Strategic Triage of Suspected Cases of Imported COVID-19
}

\author{
Hui Jial,* \\ Yuele Chang',* \\ Long Zhao' \\ Yunxia $\mathrm{Li}^{1}$ \\ Lei Chen' \\ Qian Zhang' \\ Xianzhi Lou ${ }^{2}$ \\ Chenwei $\mathrm{Li}^{2}$ \\ Shuyue Xia
}

'Department of Respiratory and Critical Care Medicine, Shenyang Central Hospital Affiliated to Shenyang Medical College, Shenyang, II 0000, People's Republic of China; ${ }^{2}$ Department of Infectious Disease, Shenyang Central Hospital Affiliated to Shenyang Medical College, Shenyang, II 0000, People's Republic of China

*These authors contributed equally to this work
Correspondence: Shuyue Xia

Department of Respiratory and Critical

Care Medicine, Shenyang Central Hospital Affiliated to Shenyang Medical College,

No. 5 Of Nanqi West Road, Tiexi

District, Shenyang, I 10000, People's

Republic of China

Tel +8613309880832

Email xiashuyuelk@I63.com
Introduction: Novel coronavirus pneumonia (COVID-19) is an acute respiratory infectious disease, which has the characteristic of human-to-human transmission and is extremely contagious. Correctly standardizing the process of early screening of infection or suspected cases in the fever clinic has become a key part of the fight against the pandemic.

Methods: A retrospective analysis of patients in the fever clinic of Shenyang Medical College Affiliated Central Hospital from January 23 to March 1, 2020, was conducted in the present study.

Results: It was found that 16 suspected cases of COVID-19 in the fever clinic were diagnosed with respiratory infections, accounting for $0.59 \%$.

Conclusion: In case of a negative result in the second nucleic acid test, strategic triage and typing might be more conducive for the following nucleic acid tests for suspected cases in order to prevent the spread of the epidemic caused by missed diagnosis.

Keywords: COVID-19, infection control, epidemic prevention and control, work process, fever clinic

\section{Introduction}

Since December 2019, the novel coronavirus pneumonia (COVID-19) epidemic in China has been testing the country's medical disease prevention and treatment system. The World Health Organization declared the outbreak as a public health emergency of international concern on January 30, 2020, and designated the outbreak as a pandemic on March 11, 2020. The disease is an acute respiratory infection caused by the novel coronavirus. ${ }^{1,2}$ It spreads via human-to-human transmission through respiratory droplets and contact ${ }^{3,4}$ and is extremely contagious. The initial symptoms of infected patients are primarily fever, fatigue, and dry cough but gradually develop into severe symptoms such as dyspnea. It has been reported that $\mathrm{CoV}$ infections could produce significant changes to multiple progress of autophagy, and autophagy could make an important role in the viral life cycle. ${ }^{5}$ Accumulating evidence suggested that the structural protein-spike (S) encode by the genomes of CoVs could make the virus to enter host cells. ${ }^{6}$ Many asymptomatic infections with positive nucleic acid results have also been found. Due to the rapid outbreak of the epidemic, there is no cure, though researchers all over the world are developing antiviral vaccines. The outpatient clinics in hospitals are important places wherein susceptible subjects may gather. Reducing the contact between suspected or confirmed patients and ordinary outpatients is a key link to protect the latter, prevent cross-infection, and stop the spread of the epidemic. Thus, the 
data of outpatient visits in our hospital from January to March 2020 were analyzed with the aim to elaborate the rationality of launching fever clinics in general hospitals during the process of receiving new cases of respiratory infectious diseases.

\section{Materials and Methods}

According to the Diagnosis and Treatment Plan of COVID-19 of the National Health Commission, ${ }^{7}$ from January 23 to March 1, 2020, in accordance with the standard of a clear or suspicious epidemiological history, a total of 2724 patients in the fever clinic of Shenyang Medical College Affiliated Central Hospital as well as a total of 4154 patients in the respiratory outpatient department were included in the study. The flow chart of the hospital visits is shown in Figure 1.

Patient classification: fever clinics and general clinics; According to the diagnostic criteria of pneumonia, suspected and confirmed fever clinics are identified as pneumonia or non-pneumonia, non-pneumonia includes other systemic diseases, such as acute gastroenteritis and chronic hepatitis B.

This paper analyzed the data of patients visiting general clinics and fever clinics in our hospital from January 2020 to March 2020, and explore the effectiveness of triage strategy which reduce cross infection.

All fever patients in the hospital were required to visit the fever clinics, and the patients who came to our hospital and planned to refer to the general clinic were selected for predictive questioning. If the patients were found to have fever, they would be required to visit the fever clinics by a special channel; the patients without fever would refer to each department for clinic treatment.

Diagnostic criteria for community acquired pneumonia were as follows: 1) Community onset. 2) (a) Increased symptoms of a recent cough, sputum or existing respiratory disease with or without chest pain/dyspnea/hemoptysis; (b) fever; (c) pulmonary consolidation signs and/or moist rales; D. $\mathrm{WBC}>10 \times 10^{9} / \mathrm{L}$ or $<4 \times 10^{9} / \mathrm{L}$ with or without left neutrophil nuclei. 3) Chest imaging examination showed patchy or patchy infiltrating shadow or interstitial changes with or without pleural effusion. Meeting any of the criteria of 1,3 and 2, and with the exception of pulmonary tuberculosis, lung tumor, non-infective interstitial lung disease, pulmonary edema, atelectasis, pulmonary embolism, pulmonary infiltration and eosinophils pulmonary vasculitis, the clinical diagnosis of community acquired pneumonia could be established. ${ }^{7}$ According to the clinical manifestations, the suspected cases were classified as follows: pneumonia type, in which there existed an opacity of viral pneumonia in the imaging examination, and non-pneumonia type, with only fever or respiratory symptoms.

Medical staff in fever clinics should strictly abide by the Law of the People's Republic of China on the Prevention and Control of Infectious Diseases and the relevant laws and regulations issued by the country during the epidemic prevention and control of infectious diseases to carry out daily disinfection management of fever clinics, isolation management of patients with fever, and protection of medical staff to avoid cross-infection. ${ }^{8}$

Nucleic acid detection: Extraction: The QIAamp Viral RNA Mini Kit (Beijing Jinhaozong Co., Ltd. Jingjing Machinery Equipment 20160028) was used, and the nucleic acid was extracted according to the instructions.

PCR amplification: Each sample was tested with the 2019-nCoV ORF1ab/N reaction solution and judged based on the test results. A negative control and positive control were set for each experiment, and both participated in the nucleic acid extraction. Detection of the amplification: The PCR amplification tubes with the loaded sample were transferred to an automatic fluorescence quantitative PCR detector for amplification detection.

\section{Results \\ Condition of the Fever Clinic Visits}

From January 23 to March 1, 2020, there were 2724 cases of fever among the clinic visits in the Affiliated Central Hospital of Shenyang Medical College, including 2287 cases of respiratory infection, 160 cases of acute gastrointestinal inflammation, 108 cases of simple fever, 104 cases of chronic hepatitis B, 18 cases of upper respiratory tract infection, 11 cases of urinary tract infection, 5 cases of bacterial enteritis, 4 cases of bacterial pneumonia, 4 cases of epidemic adenitis, and 3 cases of chickenpox. Among these patients, the cases with respiratory infection accounted for $88.7 \%$, and those with other etiologies accounted for $11.3 \%$. Among the 2287 patients with fever and respiratory infection, the 16 suspected cases with COVID-19 accounted for $0.7 \%$. All such cases were negative after two nucleic acid tests, with 6 cases of the pneumonia type and 10 cases of the non-pneumonia type. However, of the pneumonia type, one case was positive in the third nucleic acid test and confirmed with the diagnosis of COVID-19, which accounted for $6.25 \%$ of the 


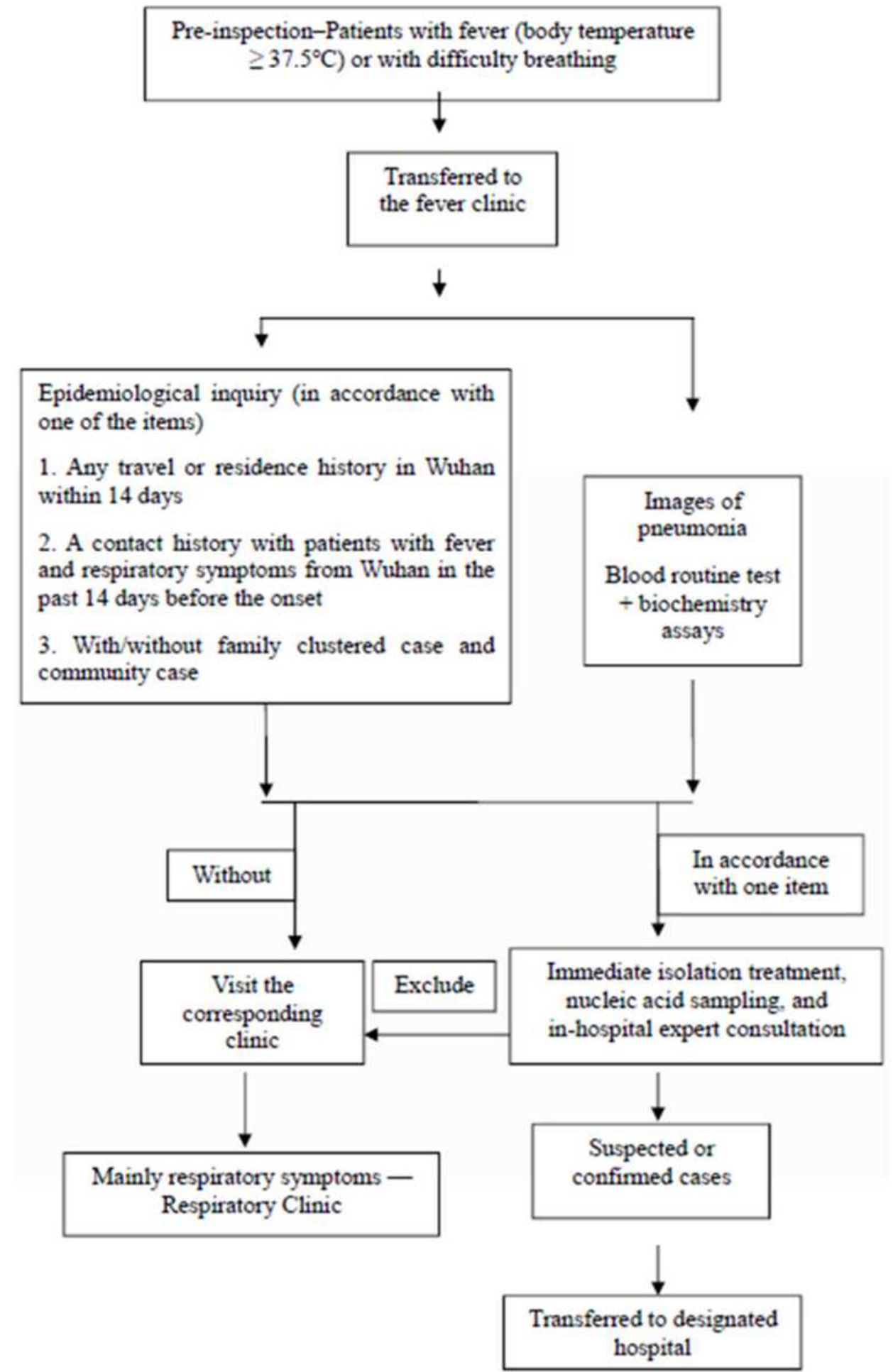

Figure I The flow chart of the visits to the hospital.

suspected cases. The case was confirmed on the third day of onset at the time of diagnosis.

During this period, the sixth version of the national diagnosis and treatment plan of COVID-19 was implemented, and of the 16 patients considered as suspected cases of COVID-19, 12 had an epidemiological history (in accordance with the sixth version of the diagnosis and treatment plan of COVID-19), accounting for $75 \%$, and 4 did not have an epidemiological history, accounting for $25 \%$. The average age of the patients was $38 \pm 12$ years, 
with a male:female ratio of 5:11. The clinical manifestations were as follows: Fever with a body temperature of $37.3^{\circ} \mathrm{C}-38.9^{\circ} \mathrm{C}$, accounting for $93.75 \%(15 / 16)$; sore throat, accounting for $37.5 \%$ (6/16); cough, accounting for $31.25 \%(5 / 16)$; and difficulty breathing, accounting for $6.25 \%(1 / 16)$. There was no diarrhea in these patients. The average time from the onset of disease to the visit was $4.06 \pm 2.38$ ( $1-9$ days).

The white blood cell count of the suspected cases at the initial visit was $(6.74 \pm 3.60) \times 10^{9} / \mathrm{L}$, the percentage of neutrophils was $68.03 \pm 12.08 \%$, and the absolute count of neutrophils was $(4.92 \pm 2.84) \times 10^{9} / \mathrm{L}$. The percentage of lymphocytes was $25.88 \pm 11.23 \%$, and the absolute count of lymphocytes was $(1.77 \pm 0.85) \times 10^{9} / \mathrm{L}$. The ratio of the absolute count of neutrophils/lymphocytes was 3.35 \pm 2.28 .

Suspected cases and results of the 14-day follow-up:

Except for the 1 confirmed case, 15 of the suspected cases tested negative for IgM and IgG during the 14-day follow-up without clinical respiratory manifestations and with normal body temperature. The details of the specific disease screening at the fever clinics are illustrated in Figure 2.

\section{Discussion}

The pre-inspection triage, as the outpost of the outpatient clinic, started after the outbreak of COVID-19 in December 2019. In accordance with the Law of the People's Republic of China on the Prevention and Control of Infectious Diseases, the hospital initially stipulated COVID-19 as a class B infectious disease according to the requirements of the management of class A infectious diseases, ${ }^{7}$ and the fever clinic process was formulated. Based on the different concepts of the epidemiological history in the Diagnosis and Treatment Plan of COVID-19 issued by the National Health Commission, a management plan for "observed cases," "suspected cases," and "confirmed cases" was formulated. We conducted a retrospective analysis of patients in the fever clinic from January 23 to March 1, 2020, and found that patients with respiratory infection accounted for $88.7 \%$ of those who went to the fever clinic. Among these patients, 16 were suspected of COVID-19 infection, accounting for $0.59 \%$. These cases were negative after two nucleic acid detection tests, with 6 cases of the pneumonia type and 10 cases of the non-pneumonia type. Among those of the pneumonia type, however, one case was positive in the third nucleic acid test and finally diagnosed with COVID-19. Therefore, it could be suggested that pre-inspection triage might play an important role in the detection of suspected cases. At the beginning of the epidemic, when little was known about COVID-19, it might have been more beneficial to divide the suspected cases into the pneumonia type and non-pneumonia type, which may have been more conducive for the close observation of suspected cases of the pneumonia type if the second nucleic acid test was negative. With the persistence of the condition, such as via fever and specific pulmonary images, it is necessary to test for a third time (or more) so as not to lead to the spread of the epidemic as a result of the missed diagnosis. Besides, laboratory parameters could also be the diagnosis for detection of COVID-19. Accumulated studies has reported that many kinds of comorbidities are relative with severe degree of COVID-19, such as diabetes mellitus, dyslipidemia, and thyroid disease. ${ }^{9-12}$ According to the an meta analysis, ${ }^{13}$ serum procalcitonin, C-reactive protein, D-dimer and LDH are higher in patients with severe COVID-19, while serum almanuin is lower than that of non-severe COVID-19 patients.

On April 1, 2020, a research team from Germany published a paper entitled "Virological assessment of hospitalized patients with COVID-2019" online in Nature, which revealed the process of the novel coronavirus invading the human body from the perspective of human virus dynamics. Nine patients from Munich were included in the study. Through the monitoring and analysis of the biological samples of the patients, it was found that the novel coronavirus rapidly replicated in the oropharynx/nasopharynx during the early stage of infection, especially within the first 5 days of the onset of symptoms. The RNA load was as high as $7.11 \times 10^{8}$ copies/swab, making it easy to be spilled out of the body and cause widespread transmission. Therefore, once a patient develops symptoms, nucleic acid testing and closed-loop management are very important. ${ }^{14}$

As of June 29, 2020, it took 13 days for the number of confirmed cases with COVID-19 in the world to increase from 1 to 2 million and only 6 days to increase from 9 to 10 million. The duration of the newly added million was the shortest since the outbreak, indicating that the spread of the epidemic is very quick. If the control is ineffective, the speed of expansion will increase as the population base increases, and the time it takes to double the number of patients will be shortened. The global epidemic of COVID-19 has not yet reached its peak and will rise in the future. ${ }^{15}$ 


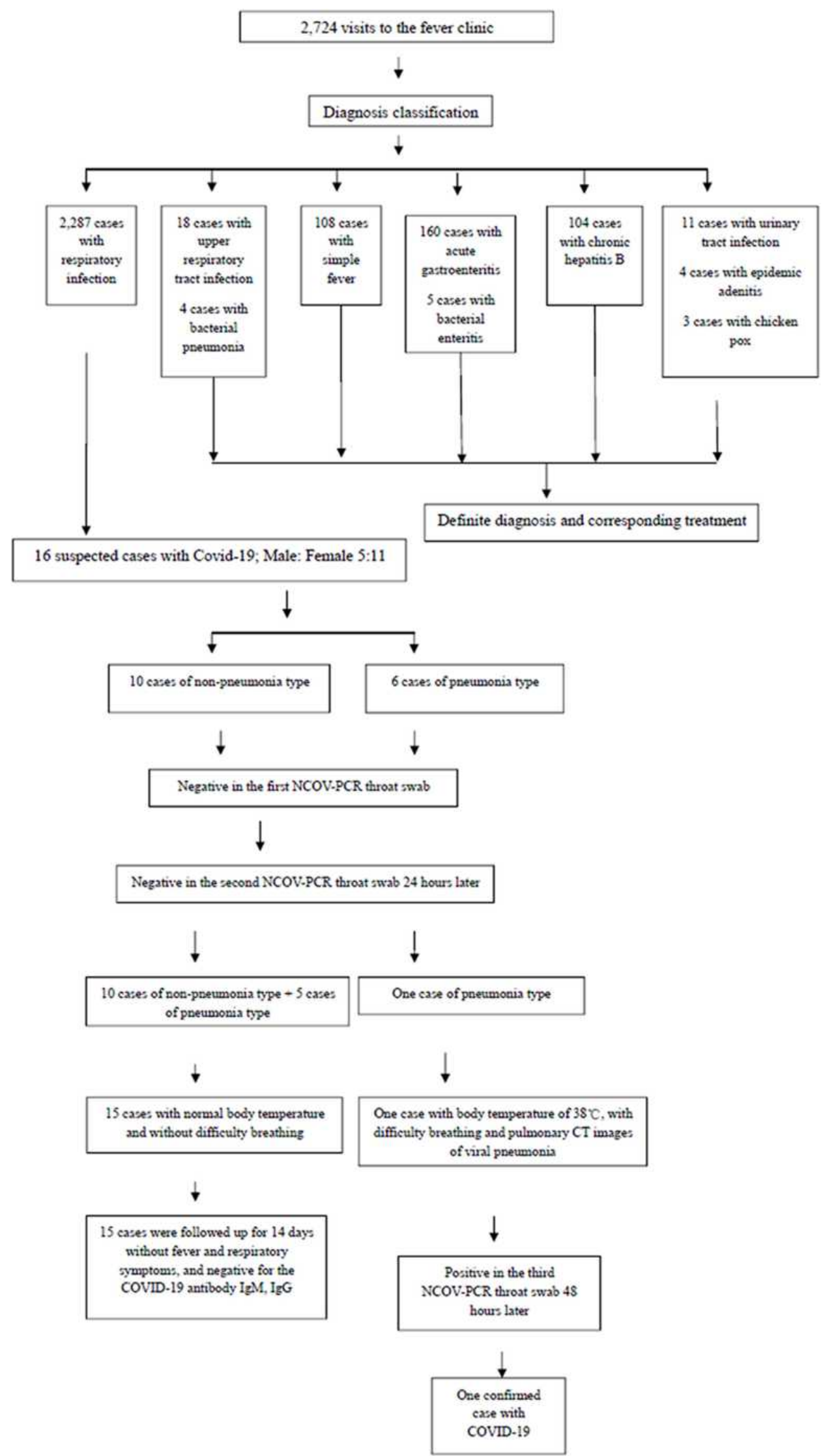

Figure 2 Disease screening chart for the fever clinic visits from January 23 to February 24, 2020. 
Nucleic acid detection is an important technique for the control of COVID-19. It can not only assist in the diagnosis of patients with symptoms but also screen in advance those who do not have symptoms. This is an essential means of finding patients and the sources of infection in time, ${ }^{16-18}$ as the establishment of scientific and reasonable pre-inspection triaging and fever clinics in a hospital might play an irreplaceable role in the early detection, early diagnosis, early reporting, and early isolation of suspected cases. Resuming work and production so as to gradually normalize national production and life while keeping the epidemic at a low level is the biggest challenge currently.

Some limitations still existed in this study. Firstly, we just analyzed the outpatients in our hospital, which is a single center and limited number of patients. Besides, as the subjects of this study was patients in clinic, it failed to systematically count the indicators, which was also another defect of this study.

\section{Conclusions}

Our study found that there is rationality and necessity of establishing fever clinics in general hospitals in the triage of new respiratory infectious diseases, which could avoid missed diagnosis and the possibility of epidemic spread.

\section{Funding}

This study was supported by the establishment of a novel coronavirus infection system for rapid triage of pneumonia and early detection of cryptogenic infections YJ20209-019.

\section{Disclosure}

The authors report no conflicts of interest in this work.

\section{References}

1. Hu B, Huang S, Yin L. The cytokine storm and COVID-19. J Med Virol. 2020. doi:10.1002/jmv.26232

2. Kannan S, Shaik Syed Ali P, Sheeza A, Hemalatha K. COVID-19 (Novel Coronavirus 2019) - recent trends. Eur Rev Med Pharmacol Sci. 2020;24(4):2006-2011. doi:10.26355/eurrev_202002_20378
3. Zu ZY, Jiang MD, Xu PP, et al. Coronavirus disease 2019 (COVID-19): a perspective from China. Radiology. 2020;296(2): E15-E25. doi:10.1148/radiol.2020200490

4. Shi Y, Wang G, Cai XP, et al. An overview of COVID-19. J Zhejiang Univ Sci B. 2020;21(5):343-360. doi:10.1631/jzus.B2000083

5. Fehr AR, Perlman S. Coronaviruses: an overview of their replication and pathogenesis. Methods Mol Biol. 2015;1282:1-23.

6. Finkel Y, Mizrahi O, Nachshon A, et al. The coding capacity of SARS-CoV-2. Nature. 2020;589:125-130. doi:10.1038/s41586-0202739-1

7. National Health and Wellness Commission. Guidelines for the Treatment of Novel Coronavirus Pneumonia (Trial 3rd-7th Edition); 2020.

8. Jia H, Peng X, Yao X, et al. Survey report on the implementation of the technical specification for hospital isolation WS/T311-2009 [j]. Chin J Infect Control. 2019;18(5):422-429.

9. Hariyanto TI, Kurniawan A. Thyroid disease is associated with severe coronavirus disease 2019 (COVID-19) infection. Diabetes Metab Syndr. 2020;14(5):1429-1430. doi:10.1016/j.dsx.2020.07.044

10. Hariyanto TI, Kurniawan A. Metformin use is associated with reduced mortality rate from coronavirus disease 2019 (COVID-19) infection. Obes Med. 2020;19:100290. doi:10.1016/j. obmed.2020.100290

11. Hariyanto TI, Kurniawan A. Statin therapy did not improve the in-hospital outcome of coronavirus disease 2019 (COVID-19) infection. Diabetes Metab Syndr. 2020;14(6):1613-1615. doi:10.1016/j.dsx.2020.08.023

12. Hariyanto TI, Putri C, Situmeang RFV, Kurniawan A. Dementia is a predictor for mortality outcome from coronavirus disease 2019 (COVID-19) infection. Eur Arch Psychiatry Clin Neurosci. 2020;26:1-3.

13. Hariyanto TI, Japar KV, Kwenandar F, et al. Inflammatory and hematologic markers as predictors of severe outcomes in COVID-19 infection: a systematic review and meta-analysis. $\mathrm{Am}$ J Emerg Med. 2021;41:110-119. doi:10.1016/j.ajem.2020.12.076. PMID: 33418211. PMCID: PMC7831442

14. Wölfel R, Corman VM, Guggemos W, et al. Virological assessment of hospitalized patients with COVID-2019. Nature. 2020;581:465-469. doi:10.1038/s41586-020-2196-x

15. Hamed MA. An overview on COVID-19: reality and expectation. Bull Natl Res Cent. 2020;44(1):86. doi:10.1186/s42269-020-00341-9

16. Sri Santosh T, Parmar R, Anand H, Srikanth K, Saritha MA. Review of salivary diagnostics and its potential implication in detection of Covid-19. Cureus. 2020;12(4):e7708. doi:10.7759/cureus. 7708

17. Harikrishnan P. Saliva as a potential diagnostic specimen for COVID-19 testing. J Craniofac Surg. 2020;31(6):e653-e655. doi:10.1097/SCS.0000000000006724.

18. Rao M, Rashid FA, Sabri FSAH, et al. Comparing nasopharyngeal swab and early morning saliva for the identification of SARS-CoV-2. Clin Infect Dis. 2020; ciaa1156.
International Journal of General Medicine

\section{Publish your work in this journal}

The International Journal of General Medicine is an international, peer-reviewed open-access journal that focuses on general and internal medicine, pathogenesis, epidemiology, diagnosis, monitoring and treatment protocols. The journal is characterized by the rapid reporting of reviews, original research and clinical studies across all disease areas. The manuscript management system is completely online and includes a very quick and fair peer-review system, which is all easy to use. Visit http://www.dovepress.com/ testimonials.php to read real quotes from published authors. 